\title{
Linear System Identifiability from Distributional and Time Series Data
}

\author{
Anandh Swaminathan ${ }^{1}$ and Richard M. Murray ${ }^{1}$
}

\begin{abstract}
We consider identifiability of linear systems driven by white noise using a combination of distributional and time series measurements. Specifically, we assume that the system has no control inputs available and can only be observed at stationarity. The user is able to measure the full stationary state distribution as well as observe time correlations for small subsets of the state. We formulate theoretical conditions on identifiability of parameters from distributional information alone. We then give a sufficient condition and an effective necessary condition for identifiability using a combination of distributional and time series measurements. We illustrate the ideas with some simple examples as well as a biologically inspired example of a transcription and degradation process.
\end{abstract}

\section{INTRODUCTION}

In this paper, we consider the combination of distributional and time series measurements in system identification. Distributional measurements consist of measuring the distribution of an output across an ensemble of systems at one given time. Time series measurements consist of measuring the output of one system over time and are standard in control theory. The central idea of this paper is that it can be advantageous for system identifiability to combine distributional and time series measurements.

In synthetic and systems biology, we are often interested in experimentally probing the dynamics of biomolecular circuits in single cells. Distributional measurements in biology such as mRNA FISH (fluorescence in situ hybridization) [1], [2] and flow cytometry allow for quantifying mRNA or protein abundance for many genes across thousands of single cells. This results in output histograms as shown in Figure 1. Time series measurements such as time lapse fluorescence microscopy [3] allow us to measure a single cell's fluorescence over time as illustrated in Figure 1. However, time lapse microscopy measurements suffer from low output dimensionality and small sample sizes when compared to distributional measurements.

Therefore, our central idea is that it can be advantageous to combine the dynamic information from time series measurements with the high dimensional information from distributional measurements when investigating system dynamics.

It is commonly known that single cells behave stochastically [4], [5]. Common biological models for stochastic single cell behavior include stochastic linear systems [6], dynamic Bayesian networks [7], [8], and stochastic chemical reaction networks [9]. While stochastic chemical reaction

This work was supported by AFOSR grant FA9550-14-1-0060. A. Swaminathan was also supported by the NSF GRFP

${ }^{1}$ The authors are with the Department of Control and Dynamical Systems, California Institute of Technology, Pasadena, CA, USA aswamina@caltech.edu

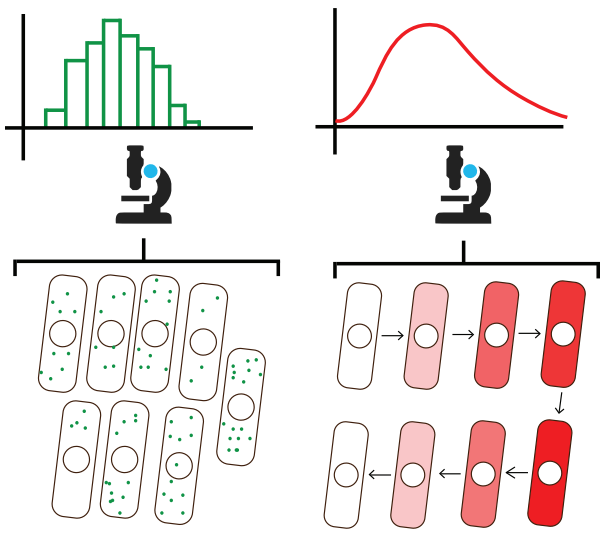

Fig. 1. Population snapshot measurements generate output histograms (left) while time series measurements generate output trajectories (right)

models are more physically relevant, for theoretical tractability, we restrict our attention in this work to linear system models, which might still accurately model equilibrium fluctuations in a stationary stochastic process [6].

Linear system identification from time series data is a very well studied problem [10]. System identification from distributional data is less common but typically arises in inference of single cell dynamics from flow cytometry measurements [11], [12], [13]. Distributional measurements in the form of sample covariances were also used in machine learning to find sparse graphical models [14], [15]. The combination of distributional and time series data for fully observable discrete time stochastic linear systems was considered computationally in [16]. The theory of dynamical structure functions has also been applied to identifiability of stochastic linear systems [17] as well as identifiability when different subsets of the state can be measured [18].

Importantly, Anderson [19] and Glover [20] considered system identifiability for stationary stochastic linear systems from output correlation measurements. In this work, we rely heavily on Anderson's and Glover's results. Our contribution is a consideration of stochastic linear system identifiability at stationarity given a combination of output correlation measurements in addition to the full steady state distribution. We term this the sensor fusion problem, because we are fusing distributional measurements with time series measurements for identification. Like Anderson and Glover, we assume that we have perfect measurements.

The structure of the paper is as follows. In Section 2, we cover some preliminaries on linear systems driven by noise and set up the sensor fusion problem we would like to solve. In Section 3, we consider the case where we have 
only distributional measurements available. In Section 4, we introduce a sufficient condition and an effective necessary condition for system identifiability in the sensor fusion case when distributional and time series measurements are available. In Section 5, we consider a detailed simple example, a more complicated toy example with decaying oscillations, and a biologically inspired example. We discuss future work and conclude in Section 6.

\section{Problem Setup}

\section{A. Preliminaries}

In this paper, we are concerned with linear systems driven by noise, and we assume that the systems have reached stationarity. We use the continuous time system description given in (1).

$$
\begin{gathered}
\dot{x}(t)=A x(t)+B w(t) \\
y(t)=C x(t) \\
\mathbf{E}\left[w(t) w(t+\tau)^{T}\right] \sim I \delta(\tau),
\end{gathered}
$$

where $A \in \mathbf{R}^{n \times n}, B \in \mathbf{R}^{n \times m}$, and $C \in \mathbf{R}^{p \times n}$ are real matrices. The state $x \in \mathbf{R}^{n}$ corresponds to physically relevant quantities, and $w(t)$ is a unit white noise process in $m$ dimensions, where $m \leq n$. The number of outputs measured is $p$, which is smaller than $n$. In this work, we assume we can choose each row of $C$ to be a canonical unit vector, so that the output is some subset of the state. We refer to $A, B B^{T}$, and $C$ as the dynamics, noise, and output matrix respectively.

The transfer function for this linear system is given by $G(s)=C(s I-A)^{-1} B$, and $(A, B, C)$ is a state space realization of $G(s)$. A minimal state space realization of $G(s)$ is one that is both observable and controllable.

We use $G^{*}$ to denote the conjugate transpose of a complex matrix $G$, and we use $A^{T}$ to denote the transpose of a real matrix $A$. We also write $P \succ 0$ or $P \succeq 0$ to mean that a matrix $P$ is positive definite or positive semidefinite respectively.

Finally, general linear systems can have a feed through term $D$ directly from input to output so that $y(t)=C x(t)+$ $D w(t)$. In this paper, we assume that $D=0$, and we will generally give state space realizations with only three matrices $(A, B, C)$. However, in some cases we will give state space realizations with four matrices. In this case, the final matrix is the feed through matrix $D$.

We then make the following assumption for the remainder of the paper.

Assumption 1: Given the linear system (1), we assume that $A$ is Hurwitz stable and that $(A, B)$ is controllable.

For a review of linear control theory, see [21].

\section{B. Distributional Measurements for Linear Systems}

The following standard result, which can be found in [21], characterizes the stationary state distribution of a linear system driven by white noise.

Proposition 1: Given Assumption 1, the stationary state distribution of the linear system (1) is a multivariate Gaussian distribution with zero mean and covariance $P$, where $P$ is positive definite and is the unique solution to (2).

$$
A P+P A^{T}+B B^{T}=0
$$

Proposition 1 allows us to assume that the distribution we observe is Gaussian. Since the form of a Gaussian distribution is fully determined by its mean and covariance and the mean is zero, we can get full knowledge of the steady state distribution from the covariance alone. Assumption 1 means that $(A, B)$ is controllable, which guarantees us a strictly positive definite covariance $P$. The Lyapunov equation (2) provides a constraint that $A$ and $B$ must satisfy in order to produce a given covariance $P$.

\section{Time Series Measurements for Linear Systems}

Time series measurements allow us to measure output trajectories. There are two main types of time series measurements depending on whether a controllable input to the system is available. We start with the more standard case.

1) Time Series Measurements with Controlled Input: If we consider $w(t)$ to be a controllable input in (1), then we can characterize the input output transfer function $G(s)$ of the linear system given in (1). The following standard result characterizes the space of state space realizations consistent with a transfer function. Again, see [21] for more information.

Proposition 2: Given a transfer function $G(s)$ with a minimal realization given by $(A, B, C)$, then $(\hat{A}, \hat{B}, \hat{C})$ is also a minimal realization of $G(s)$ if and only if

$$
\hat{A}=T A T^{-1} \quad \hat{B}=T B \quad \hat{C}=C T^{-1}
$$

for an invertible transformation $T$.

Proposition 2 tells us that the state space matrices can only be resolved up to a change of coordinates. This only holds if the system is of minimal order.

2) Output Correlation Measurements: When the input is an unobservable noise as in (1), we can only measure the system output, so we cannot expect to recover the input output transfer function of the system. However, we can observe correlations in the output, or equivalently the output spectral density.

Given the linear system (1), we can measure the time correlations in the output $y$. Since the process is at stationarity, this corresponds to measuring the correlation function $R(\tau)=\mathbf{E}\left[y(t+\tau) y(t)^{T}\right]$. In the frequency domain, the spectral density is given by

$$
\Phi(s)=G(s) G^{*}(s),
$$

where

$$
G(s)=C(s I-A)^{-1} B
$$

is the input output transfer function of the system.

The problem of finding state space matrices that generate a specified spectral density has been studied extensively by Anderson [19] and Glover [20]. We present some of their results here. 
We first define the notion of a globally minimal system as done in [19] and [20].

Definition 1: A minimal state space system $(A, B, C)$ is globally minimal with respect to a spectral density function $\Phi(s)$ if $G(s)=C(s I-A)^{-1} B$ satisfies $\Phi(s)=G(s) G^{*}(s)$ and $A$ has the smallest dimension possible for any solution to $\Phi(s)=G(s) G^{*}(s)$.

Specifically, a minimal system can sometimes produce an output spectral density that can be reproduced by another minimal system of lower order. In this case, the original system is minimal but not globally minimal. This scenario occurs when the original transfer function contains an all pass component, which is a term like $\frac{s-a}{s+a}$ that cancels out when forming the spectral density. We make the following assumption with respect to output correlation measurements.

Assumption 2: Given the linear system (1), we assume that we know the system order $n$ a priori, that $A$ is Hurwitz stable, and that $(A, B, C)$ is a globally minimal realization for the transfer function $G(s)=C(s I-A)^{-1} B$ for any choice of $C$ that we use.

Assumption 2 is strictly stronger than Assumption 1. Both loss of controllability and pole zero cancellation are unlikely occurences within the space of all linear systems, so our hope is that these assumptions are not too restrictive. The following lemma from [19] characterizes the set of globally minimal systems consistent with a spectral density.

Lemma 1: Given an output spectral density of $\Phi(s)$, let $\Phi(s)=Z(s)+Z^{*}(s)$, where $Z(s)$ is positive real. Then, suppose $(A, G, C, J)$ is a minimal realization for $Z(s)$. Consider the following LMI (4).

$$
\left[\begin{array}{cc}
A P+P A^{T} & P C^{T}-G \\
C P-G^{T} & -J-J^{T}
\end{array}\right]=-\left[\begin{array}{l}
B \\
D
\end{array}\right]\left[\begin{array}{ll}
B^{T} & D^{T}
\end{array}\right]
$$

Then all globally minimal realizations of $G(s)$ such that $\Phi(s)=G(s) G^{*}(s)$ have a realization given by $(A, B, C, D)$ where $B$ and $D$ satisfy (4) together with $P \succ 0$. Also, if $B$ and $D$ together with $P \succ 0$ satisfy (4), then $G(s)=$ $C(s I-A)^{-1} B+D$ is a globally minimal solution of $G(s) G^{*}(s)=\Phi(s)$.

Lemma 1 characterizes all globally minimal state space realizations consistent with the output spectral density. In addition, the minimal dimension of the positive real transfer function matrix $Z(s)$ sets the globally minimal dimension for the system. The following result from [20] describes the relationship between multiple globally minimal realizations.

Lemma 2: If $\left(A_{1}, B_{1}, C_{1}, D_{1}\right)$ is a globally minimal realization for $\Phi(s)$, then $\left(A_{2}, B_{2}, C_{2}, D_{2}\right)$ is also a globally minimal realization if and only if there is an invertible $T$ and symmetric $Q$ such that

$$
\begin{aligned}
A_{1} & =T A_{2} T^{-1} \\
C_{1} & =C_{2} T^{-1} \\
Q A_{1}^{T}+A_{1} Q & =-B_{1} B_{1}^{T}+T B_{2} B_{2}^{T} T^{T} \\
Q C_{1}^{T} & =-B_{1} D_{1}^{T}+T B_{2} D_{2}^{T} \\
D_{1} D_{1}^{T} & =D_{2} D_{2}^{T} .
\end{aligned}
$$

Lemma 2 gives us an algebraic condition that relates globally minimal system realizations to each other. It is clear by letting $Q=0$ that a change of coordinates is sufficient to satisfy the conditions of Lemma 2, meaning that the space of consistent systems is at least as large as in the controlled input case. This makes it mathematically clear why a controlled input is superior for identification.

\section{Sensor Fusion Problem}

We then set up the identifiability problem when both distributional and time series measurements are available. We assume we have a linear system of a known minimal order $n$ that may be parametrized. However, we assume no parametrization for our results. We are interested in recovering the dynamic matrix $A$ and the noise matrix $B B^{T}$.

We know the matrix $C$, because we already know which system states we are measuring. This corresponds to measuring a certain protein concentration in a cell or the value of a node in a network. We also might measure multiple subsets of the state in separate experiments using different matrices $C_{1}, C_{2}$, et cetera. For each choice of $C$, we assume we can measure the associated output correlation function. We also assume that we can measure the stationary state covariance $P$. Furthermore, we assume that all measurements are perfect measurements with zero error.

With this information, we would like to assess system identifiability both using distributional measurements alone as well as using a combination of distributional and time series measurements.

\section{IDENTIFIABILITY USING COVARIANCE MEASUREMENTS ONLY}

In this section, we consider the set of dynamic matrices $A$ that are consistent with a measured covariance matrix $P$. We assume in this section that the noise matrix $B B^{T}$ is known as well. The following propositions are straightforward linear algebra results.

Proposition 3: Define the linear transformation $L_{P}(A)=$ $A P+P A^{T}$. The transformation $L_{P}$ takes $n \times n$ real matrices to $n \times n$ symmetric matrices. Then, consider the linear system (1) with $B$ given. Then, the linear system (1) satisfies Assumption 1 and has a steady state covariance of $P \succ 0$ if and only if $A=-\frac{1}{2} B B^{T} P^{-1}+N$ with $N \in \operatorname{ker} L_{P}$ and $A$ is Hurwitz or equivalently $(A, B)$ is controllable.

Proof: Since $L_{P}$ is a linear transformation that takes $n \times n$ matrices to $n \times n$ symmetric matrices, we can write down all solutions to the continuous time Lyapunov equation (2) by using the particular solution $-\frac{1}{2} B B^{T} P^{-1}$ and adding a term from the kernel. However, this does not guarantee $A$ must be Hurwitz, so we add that condition in separately. Since $P \succ 0$, the controllability of $(A, B)$ is also sufficient to imply that $A$ is Hurwitz [21].

The space where $A$ is not Hurwitz and rather marginally stable is generally a set of measure zero within the affine subspace of $\mathbf{R}^{n \times n}$ given by $-\frac{1}{2} B B^{T} P^{-1}+\operatorname{ker} L_{P}$. Furthermore, it is easy to see that $L_{P}$ is surjective onto the 
space of symmetric matrices and therefore, by rank nullity theorem, the dimension of $\operatorname{ker} L_{P}$ is $\frac{n(n-1)}{2}$.

This result is quite different in discrete time, and we briefly present it here for completeness.

Proposition 4: Consider the discrete time linear system $x_{k+1}=A x_{k}+B w_{k}$, where $w_{k}$ is a unit normal random variable of appropriate dimension. Also, fix $B$ and $P$ so that $P \succ 0$ and $P \succeq B B^{T} \succeq 0$. Then, the system is stable with steady state covariance $P$ if and only if $A=(P-$ $\left.B B^{T}\right)^{1 / 2} U P^{-1 / 2}$, where $U U^{T}=I$ and $A$ is Schur stable or equivalently $(A, B)$ is controllable.

Proof: The steady state covariance for discrete time systems satisfies the Lyapunov equation $A P A^{T}-P+B B^{T}=0$ [21]. In the forward direction, $A$ must be Schur stable, and $A$ must satisfy the Lyapunov equation. If $A$ satisfies $A P A^{T}-P+B B^{T}=0$, then let $F=A P^{1 / 2}$ and let $G=\left(P-B B^{T}\right)^{1 / 2}$. Then $F F^{T}=G G^{T}$ and applying Lemma 3 in [22] gives that $F=G U$ must hold, which gives the expression for $A$. The matrix $A$ must then be Schur stable by assumption and $(A, B)$ must be controllable since $A$ is Schur stable and $P \succ 0$ [21].

In the reverse direction, substitution reveals that $A$ satisfies the discrete time Lyapunov equation. If $(A, B)$ is controllable, then the fact that $P \succ 0$ implies $A$ is Schur. Then, the fact that $A$ is Schur in addition to the fact that $A$ satisfies the Lyapunov equation means that the steady state covariance will be $P$.

These propositions show that neglecting strict stability of $A$, we can resolve the $A$ matrix up to an affine subspace in continuous time and and up to an orthogonal degree of freedom in discrete time. If we can control $B B^{T}$, then multiple covariance measurements for different values of $B B^{T}$ in continuous time may resolve $A$ exactly. In discrete time, the associated Lyapunov equation is quadratic and thus we can only ever resolve $A$ up to a choice of sign.

As an example, consider the discrete time dynamics given by $x_{k+1}=A x_{k}+w_{k}$, with

$$
A=\left[\begin{array}{ccccc}
0 & 0 & 0 & 0 & 1 / 2 \\
1 / 2 & 0 & 0 & 0 & 0 \\
0 & 1 / 2 & 0 & 0 & 0 \\
0 & 0 & 1 / 2 & 0 & 0 \\
0 & 0 & 0 & 1 / 2 & 0
\end{array}\right]
$$

These dynamics are that of a damped oscillation where the state moves from one entry to the next in a damped fashion. Since $w_{k}$ has covariance $I$, we can compute a steady state covariance of $P=4 / 3 I$. Applying Proposition 4 shows that then $A=1 / 2 U$, where $U$ is orthogonal. If we are interested in obtaining the structure of $A$, steady state covariance information does not help since $A$ can be any orthogonal matrix. Furthermore, even if we know a priori that $A$ might be sparse, $A$ can still be any permutation matrix scaled by $1 / 2$, so it still does not help recover the structure of $A$.

\section{SENSOR FUSION}

As stationary distribution information alone is inadequate, we now consider the combination of distribution and time series measurements. We first give a simple sufficient condition for identifiability of the dynamic matrix from noise driven measurements. Recall that the output matrix $C$ allows us to measure a small number of elements of the state.

Proposition 5: Assume the conditions of Assumption 1. Given a collection of time series measurements with different $C$ matrices selected as $C_{1}, C_{2}, C_{3}, \ldots$, if each pair of states is measured together at least once, then the dynamic matrix can be identified exactly.

Proof: The proof follows from the autocovariance function. If all pairs of states are observable together, we can reconstruct the system's autocovariance function $R(\tau)=$ $\mathbf{E}\left[x(t+\tau) x(t)^{T}\right]$. We can calculate the derivative of the autocovariance function at zero as $\bar{R}=\left.\frac{d R}{d \tau}\right|_{\tau=0}=A P$. We also know that $R(0)$ is the steady state covariance $P$. We can recover $A=\bar{R} R(0)^{-1}$.

Proposition 5 gives us a sufficient but highly conservative condition for the use of output correlation measurements to infer the system dynamics. If we have a system with 10 states and can simultaneously measure 2 outputs at a time, then we would need to conduct $\left(\begin{array}{c}10 \\ 2\end{array}\right)$ or 45 experiments to guarantee that we could resolve the system exactly.

In the sequel, we develop an effective necessary condition that requires far fewer experiments. To do this, first of all we extend Lemma 1 to the sensor fusion setting. We start with the following lemma.

Lemma 3: Given $G(s)$ with strictly proper realization $(A, B, C)$, the positive real transfer function $Z(s)$ as defined in Lemma 1 must be strictly proper.

Proof: From [20], we know that $Z(s)$ is the Laplace transform of the autocovariance function $R_{y}(\tau)$ of the output. We know that $R_{y}(0)=C P C^{T}$ is finite and that $R_{y}(\tau)$ exponentially decays to zero since the original system is strictly proper. Therefore, the Laplace transform $Z(s)$ exists for all non-negative $s$. Then, by applying the initial value theorem, we know that $Z(s)$ goes to zero as $s$ goes to infinity so $Z(s)$ must be strictly proper.

We can then prove the following theorem.

Theorem 1: Assume the conditions of Assumption 2. Given a steady state covariance $P$ and an output spectral density of $\Phi(s)$, let $\Phi(s)=Z(s)+Z^{*}(s)$, where $Z(s)$ is positive real. Then, apply Lemma 3 and suppose $(\hat{A}, \hat{G}, \hat{C})$ is a strictly proper minimal realization for $Z(s)$. Then consider the following equation (6).

$$
\begin{gathered}
A P+P A^{T}+B B^{T}=0 \quad P C^{T}=T \hat{G} \\
A=T \hat{A} T^{-1} \quad C T=\hat{C}
\end{gathered}
$$

Then $(A, B, C)$ is a globally minimal system with output spectral density $\Phi(s)$ and steady state covariance $P$ if and only if $(A, B, C)$ satisfies (6).

Proof: The proof is a straightforward extension of Lemma 1, where we can set $J=D=0$. In the reverse direction, if (6) holds, then the top left expression gives 
a covariance of $P$ immediately. Then, define $G=T \hat{G}$ and note that $(A, G, C)$ is a coordinate transformation of $(\hat{A}, \hat{G}, \hat{C})$, so $(A, G, C)$ is a valid realization for $Z(s)$. The reverse direction of Lemma 1 guarantees the desired output spectral density. In the foward direction, by global minimality we know that $\hat{A}$ from our realization for $Z(s)$ must be a coordinate transformation $T$ away from the true $A$. Then, a different realization for $Z(s)$ with the true $A$ is given by $(A, G, C)$ with $G=T \hat{G}$ and $C=\hat{C} T^{-1}$. The foward direction of Lemma 1 implies that $P C^{T}=T \hat{G}$ and $A P+P A^{T}+B B^{T}=0$ for some $P \succ 0$. However, that last equation is the covariance equation and so it must hold for the given covariance $P$.

Theorem 1 fully characterizes the space of systems consistent with a combination of a steady state covariance and one output correlation. We consider the system identifiable if we can resolve $A$ and $B B^{T}$ uniquely from this information. We can search for a solution to the equations in Theorem 1 by using the coordinate transformation $T$ and the noise matrix $B B^{T}$ as variables. We can extend this result to the case where we measure the steady state covariance along with multiple different combinations of states as outputs. We first prove the following lemma.

Lemma 4: Assume that $A \in \mathbf{R}^{n \times n}$ has no eigenvalues with multiplicity greater than one. It follows for invertible transformations $T_{1}$ and $T_{2}$ that $T_{1} A T_{1}^{-1}=T_{2} A T_{2}^{-1}$ if and only if $T_{1}=T_{2} S \Lambda S^{-1}$, where $S$ is a matrix whose columns are eigenvectors of $A$, and $\Lambda$ is an invertible diagonal matrix. $\Lambda$ must have real entries corresponding to real columns of $S$ and complex conjugate entries corresponding to complex conjugate columns of $S$.

Proof: Multiplying the equality by the appropriate quantities, we get $T_{2}^{-1} T_{1} A=A T_{2}^{-1} T_{1}$. This holds if and only if $A$ and $T_{2}^{-1} T_{1}$ are simultaneously diagonalizable. Since $A$ has no duplicate eigenvalues, the eigendirections are fixed, so we can write $T_{1}=T_{2} S \Lambda S^{-1}$, where $S$ contains the eigenvectors of $A$. The matrix $\Lambda$ must have nonzero real entries corresponding to real columns of $S$ and nonzero complex conjugate entries corresponding to complex conjugate columns of $S$. This is because $T_{1}$ and $T_{2}$ must be real. In the reverse direction, substitution shows $T_{1} A T_{1}^{-1}=T_{2} A T_{2}^{-1}$.

Lemma 4 tells us that if two similarity transformations $T_{1}$ and $T_{2}$ both transform a matrix $A$ to the same final matrix, then given $T_{1}$, we have only $n$ degrees of freedom when choosing $T_{2}$. This is because we have one real degree of freedom for each real eigenvector and one complex degree of freedom for each pair of complex eigenvectors. We can then formulate an effective necessary condition.

Let Assumption 2 hold and also assume that the dynamic matrix $A$ has no duplicate eigenvalues. Suppose that we are given a steady state covariance $P \succ 0$ and a set of $k$ output correlation measurements obtained with $k$ different $C$ matrices $C_{1}$ through $C_{k}$. Then, we would like to solve for the true $A$ and $B B^{T}$ matrices of the system.

Using the observed spectral densities $\Phi_{i}(s)$ where $1 \leq i \leq$ $k$, we can compute each $Z_{i}(s)$ and find an $\left(\hat{A}_{i}, \hat{G}_{i}, \hat{C}_{i}\right)$ that realizes each $Z_{i}(s)$. By Assumption 2, all the $\hat{A}_{i}$ matrices will have the same dimension and be similar, so we can transform coordinates so that $\hat{A}_{i}=\hat{A}$ for all $i$. We then know that the true $A$ matrix is given by $T_{1} \hat{A} T_{1}^{-1}$. The other $T_{i}$ matrices must also transform $\hat{A}$ to $A$ and are forced by Lemma 4 to satisfy the equation $T_{i}=T_{1} S \Lambda_{i} S^{-1}$ for $i>1$, where $S$ contains the eigenvectors of $\hat{A}$. Then, we can write down a system of equations in terms of only $T_{1}, B B^{T}$, and $\Lambda_{i}$ where $i>1$.

Combining this with Theorem 1, we can rewrite the equations as

$$
\begin{gathered}
T_{1} \hat{A} T_{1}^{-1} P+P\left(T_{1} \hat{A} T_{1}^{-1}\right)^{T}+B B^{T}=0 \\
P C_{1}^{T}=T_{1} \hat{G}_{1} \quad C_{1} T_{1}=\hat{C}_{1} \\
P C_{i}^{T}=T_{1} S \Lambda_{i} S^{-1} \hat{G}_{i} \quad C_{i} T_{1} S \Lambda_{i} S^{-1}=\hat{C}_{i} \quad \forall i>1 .
\end{gathered}
$$

This gives us a total of $\frac{n(n+1)}{2}+2 k p n$ equations. We have $\frac{n(n+1)}{2}$ variables for $B B^{T}, n^{2}$ variables for $T_{1}$, and an additional $(k-1) n$ variables for the $\Lambda_{i}$ matrices. This gives a total of $\frac{n(n+1)}{2}+n^{2}+(k-1) n$ variables. Then, in order to have more equations than variables, we get the following constraint on the number of measurements $k$.

$$
k \geq \frac{n-1}{2 p-1}
$$

This inequality (8) is our effective necessary condition. This is not a true necessary condition because nonlinear equations in high dimensions can have unique solutions while having far fewer constraints than variables. For example, two hyper spheres can be tangent at exactly one point. Also, if $B B^{T}$ is not strictly positive definite, the positive semidefiniteness of $B B^{T}$ may also help solve for $A$ and $B B^{T}$ if for example, the solution set of some subset of the equations is tangent to the positive semidefinite cone at exactly one point. However, we typically do expect to need at least as many constraints as variables to solve a system of equations.

There is no guarantee at all that the constraints generated from multiple experiments and equations will be independent, so more experiments might be required. In the case of ten nodes and two simultaneous outputs from before, condition (8) requires three measurements.

\section{EXAMPLES}

We now consider examples of sensor fusion.

\section{A. Two Dimensional System}

In this section, we consider sensor fusion for a two dimensional system. This example leads to a negative result and an associated conjecture. We consider the system given by

$$
\begin{aligned}
A & =\left[\begin{array}{ll}
-1 & 1 / 2 \\
1 / 2 & -1
\end{array}\right] \\
B B^{T} & =\left[\begin{array}{ll}
0 & 0 \\
0 & 1
\end{array}\right] \\
C & =\left[\begin{array}{ll}
1 & 0
\end{array}\right] .
\end{aligned}
$$


In this system, we also assume that we have prior knowledge that the noise enters the system only from the second state.

Using the Lyapunov equation (2), we compute a steady state covariance

$$
P=\left[\begin{array}{cc}
\frac{1}{12} & \frac{1}{6} \\
\frac{1}{6} & \frac{7}{12}
\end{array}\right]
$$

The output spectral density of this system is given by $\Phi(s)=\frac{4}{16 s^{4}-40 s^{2}+9}$.

Then, we can apply Theorem 1 to compute the set of $A$ and $B B^{T}$ matrices consistent with the given $P$ as well as the output spectral density of the system.

Using partial fraction decomposition, we can solve for $Z(s)$, and we find

$$
Z(s)=\frac{\frac{1}{8}}{s+\frac{1}{2}}-\frac{\frac{1}{24}}{s+\frac{3}{2}} .
$$

This verifies that the globally minimal system dimension is two, and we can write down a state-space realization for $Z(s)$ as $(\hat{A}, \hat{G}, \hat{C})$ below.

$$
\hat{A}=\left[\begin{array}{cc}
-2 & -\frac{3}{4} \\
1 & 0
\end{array}\right] \quad \hat{G}=\left[\begin{array}{l}
1 \\
0
\end{array}\right] \quad \hat{C}=\left[\begin{array}{ll}
\frac{1}{12} & \frac{1}{6}
\end{array}\right]
$$

We can solve for all possible globally minimal systems consistent with this output correlation using Lemma 2. Using the true system as one globally minimal system, we solve for all other possible systems. First of all, the constraint $Q C_{1}^{T}=$ 0 implies that $Q$ is all zeros except in the bottom right corner. Then,

$$
A_{1} Q+Q A_{1}^{T}+B_{1} B_{1}^{T}=\left[\begin{array}{cc}
0 & \frac{q}{2} \\
\frac{q}{2} & 1-2 q
\end{array}\right],
$$

where $q$ is the bottom right entry of $Q$. Since this term must be positive semidefinite, then $q=0$ must hold, and we see that $Q=0$. Thus, the equations simplify down to $A_{1}=$ $T A_{2} T^{-1}, C_{1}=C_{2} T^{-1}$, and $B_{1} B_{1}^{T}=T B_{2} B_{2}^{T} T^{T}$. We can solve these equations assuming $T$ is a free two by two matrix and assuming that $B_{2} B_{2}^{T}$ is a symmetric matrix with all zeros and one positive entry in the lower right hand corner.

Then, solving the equations gives us the following expressions for the set of consistent $A_{2}$ and $B_{2} B_{2}^{T}$ matrices for this system.

$$
\begin{aligned}
A_{2} & =\left[\begin{array}{cc}
\frac{c}{2}-1 & \frac{d}{2} \\
-\frac{c^{2}-1}{2 d} & -\frac{c}{2}-1
\end{array}\right] \\
B_{2} B_{2}^{T} & =\left[\begin{array}{ll}
0 & 0 \\
0 & \frac{1}{d^{2}}
\end{array}\right] \\
c, d & \in \mathbf{R} \\
d & \neq 0
\end{aligned}
$$

Time series measurements along with knowledge of the noise structure do not provide much information about the structure of $A$ in this case. Of course, the steady state distribution is also insufficient to determine $A$ as shown in Proposition 3.

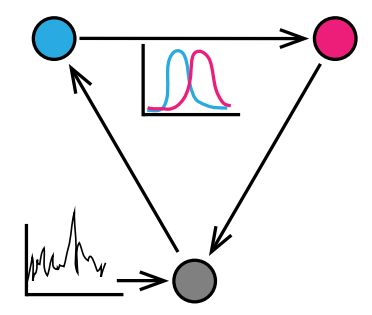

Fig. 2. Three node "oscillating" system with one noise input and two measured outputs

The next step is to consider sensor fusion. In the sensor fusion case, we solve the equations given in Theorem 1. These equations can again be solved by letting $T$ be a free variable. In this case, the noise matrix $B B^{T}$ can be found exactly. However, the $A$ matrix has two possible solutions. The first solution is the correct $A$ matrix which leads to the transfer function $G(s)$ above. The other solution is

$$
A_{\text {wrong }}=\left[\begin{array}{cc}
1 & -\frac{1}{2} \\
\frac{15}{2} & -3
\end{array}\right]
$$

which leads to a transfer function of $-G(s)$, which of course reproduces the same spectral density $\Phi(s)$ but also reproduces the same covariance at steady state. With sensor fusion, there are exactly two consistent $A$ matrices.

Interestingly, this result seems to hold for different ouput choices. For example, we could measure the second state instead of the first state or the difference of the two states. They all produce either the true $A$ matrix or the incorrect

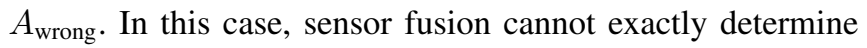
$A$, but it does provide us with two specific possible values of $A$ instead of the one or two dimensional infinite space that we get from using distribution or time series measurements alone.

\section{B. Cyclic Dynamics}

In this example, we investigate sensor fusion for a three node decaying oscillation system.

We try a few different cases in terms of which nodes are measured in the system. We start with the three dimensional system given by

$$
A=\left[\begin{array}{ccc}
-1 & 0 & 1 / 2 \\
1 / 2 & -1 & 0 \\
0 & 1 / 2 & -1
\end{array}\right]
$$

with noise entering only from the third state, so that

$$
B B^{T}=\left[\begin{array}{lll}
0 & 0 & 0 \\
0 & 0 & 0 \\
0 & 0 & 1
\end{array}\right]
$$

The system is shown in Figure 2 with the first and second nodes measured together.

The previous section just showed that measuring steady state covariance and a single output correlation is not sufficient to recover system dynamics for a two dimensional system. This limitation of single output measurements still 


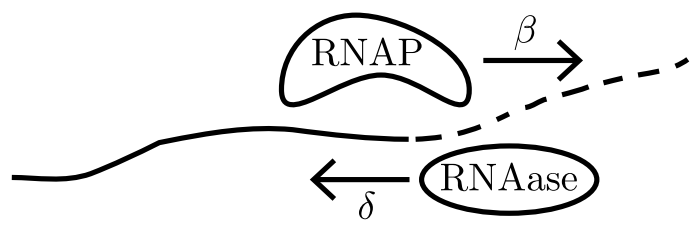

Fig. 3. Transcription and Degradation Process for mRNA

holds for this system. We then consider the case of measuring two outputs simultaneously. This is the situation illustrated in Figure 2. Noise enters into state three while states one and two are measured simultaneously.

In this case, the algebra associated with Lemma 2 leads to another messy expression for the space of possible $A$ matrices consistent with output correlations alone.

Incorporating the steady state information in this case again leads to two possible $A$ matrices while recovering $B B^{T}$ exactly. One of course is the correct $A$, while the other is given by

$$
A_{\text {wrong }}^{12}=\left[\begin{array}{ccc}
3 & -\frac{31}{6} & -\frac{1}{2} \\
\frac{1}{2} & -1 & 0 \\
\frac{161}{6} & -\frac{251}{6} & -5
\end{array}\right] .
$$

If we measure the first and third states together, then we again get two possibilities for $A$, where the incorrect one is given by

$$
A_{\text {wrong }}^{13}=\left[\begin{array}{ccc}
-1 & 0 & \frac{1}{2} \\
-\frac{65}{62} & 1 & \frac{12}{31} \\
\frac{508}{31} & -\frac{63}{2} & -3
\end{array}\right] .
$$

From these results, it is clear that if we measure steady state covariance information along with output correlations for the first and second state together as well as output correlations for the first and third state together, we can resolve $A$ exactly.

Interestingly, if we measure the output correlations for states two and three together, then that alone is sufficient when combined with the steady state covariance to resolve $A$ exactly. That is, we can resolve $A$ exactly without ever measuring state one in time series. Additionally, it is clear that identifiability is a question of which nodes we measure in addition to how many nodes we measure. This suggests a consideration of graph structure and identifiability in the same vein as well known graph theoretic analyses of controllability and observability [23].

\section{Example - RNA Elongation and Degradation}

In this section, we consider the biologically inspired example of a transcription degradation system at equilibrium. RNA Polymerase is an enzyme that transcribes and lengthens the RNA molecule polymer while RNAase is an enzyme that degrades and shortens the RNA. We illustrate this system in Figure 3 and model it using the dynamics in (10).

$$
\begin{array}{r}
\dot{x}=b+A x+B w \\
x, b, B \in \mathbf{R}^{n} \\
A \in \mathbf{R}^{n \times n}
\end{array}
$$

Here, the elements of $x$ correspond to varying lengths of partial RNA where $x(1)$ is a nascent transcript and $x(n)$ is a fully completed transcript. The rate matrix

$$
A=\left[\begin{array}{ccccc}
-\left(\delta_{1}+\beta_{1}\right) & \delta_{2} & & & \\
\beta_{1} & -\left(\delta_{2}+\beta_{2}\right) & \ddots & & \\
& \beta_{2} & \ddots & \delta_{n-1} & \\
& & \ddots & -\left(\delta_{n-1}+\beta_{n-1}\right) & \delta_{n} \\
& & & \beta_{n-1} & -\delta_{n}
\end{array}\right]
$$

is a tridiagonal matrix where the terms represent the local rates of elongation and degradation. Namely, the superdiagonal terms $\delta_{k}$ represent the local rates of degradation at different points along the transcript, the subdiagonal terms $\beta_{k}$ represent the local rates of elongation at different points along the transcript, and the diagonal terms enforce conservation. Note that the $\delta_{k}$ 's and $\beta_{k}$ 's must be positive. As constructed, $A$ is diagonally dominant and thus Hurwitz.

We model the actual transcription and degradation processes as proceeding deterministically with noise being introduced only by initiation events. The bias term $b=$ $\left[\begin{array}{llll}b_{1} & 0 & \cdots & 0\end{array}\right]^{T}$ with $b_{1}>0$ gives the mean amount of transcription initiation activity. The noise term $B=$ $\left[\begin{array}{llll}1 & 0 & \cdots & 0\end{array}\right]^{T}$ is the noise in the transcription initiation process.

We assume that we can measure the steady state distribution of this system, which we might do by looking at single cell RNA data across a population of cells. Because of the bias term, we would not only be able to measure the covariance at steady state, but we could also measure the mean $x_{e q}$, where $A x_{e q}+b=0$ must hold at steady state.

There are a total of $2 n+1$ total parameters to identify. The rate matrix $A$ has $2 n-1$ parameters, and the noise and bias term have one parameter each. Using the steady state mean information alone gives only the equation $A x_{e q}+b=0$. This only provides $n$ linear equations, so we cannot solve for all $2 n+1$ parameters.

However, using the steady state distribution gives us more information. First of all, it is clear from the structure of $A$ and $B$ that $(A, B)$ will be almost certainly be controllable as the noise can propagate to every state and therefore, the steady state covariance $P$ will be strictly positive definite and unique. Knowing the covariance provides us with another $\frac{n(n+1)}{2}$ equations through the continuous time Lyapunov equation (2).

However, since all the equations are linear, we can only determine the parameters up to multiplication by a positive scalar. Intuitively, this is because rescaling time does not affect steady state measurements. Therefore, without some additional information, we still cannot determine $A$ exactly.

For a specific numerical example, we set 


$$
A=\left[\begin{array}{cccc}
-2 & 1 & 0 & 0 \\
1 & -2 & 1 & 0 \\
0 & 1 & -2 & 1 \\
0 & 0 & 1 & -1
\end{array}\right],
$$

and we set $b=B=\left[\begin{array}{llll}1 & 0 & 0 & 0\end{array}\right]^{T}$. In this example, steady state measurements constrain $A$ to a one dimensional subspace of $\mathbf{R}^{n \times n}$. The set of dynamics consistent with the steady state mean and covariance is given by $k A$ where $k>0$. Note that $k<0$ is impossible because it would make $A$ not Hurwitz. The set of consistent $B$ matrices is given by $k B$.

Then, suppose we measure the output correlation with $C=\left[\begin{array}{llll}0 & 0 & 0 & 1\end{array}\right]$, which is the state corresponding to fully completed RNA molecules. This results in a spectral density of

$$
\Phi(s)=\frac{1}{s^{8}-19 s^{6}+87 s^{4}-70 s^{2}+1} .
$$

Since we know the true $A$ and $B$ matrices up to a scalar factor $k$, the set of spectral densities consistent with the distributional information is given by $\Phi\left(\frac{s}{k}\right)$. We see that $k=1$ must hold, which means that sensor fusion is sufficient to resolve $A, b$, and $B$ exactly for this example.

\section{CONCLUSION AND Future Work}

In this paper, we considered the idea of combining full state covariance information together with limited output correlations to improve identifiability of stationary linear stochastic systems driven by white noise. This problem was motivated by the biological setting, where often distributional measurements are high dimensional and time series measurements are low dimensional. We formulated conditions for identifiability using distributional measurements alone and also for using sensor fusion.

Future work includes developing a practical identifiability algorithm for sensor fusion that is robust to noise. This algorithm will complement the theoretical identifiability analysis developed in this paper. We then hope to demonstrate both sets of techniques on data generated from either a simulated or experimental stochastic biological circuit to demonstrate the utility of the methods.

\section{ACKNOWLEDGMENTS}

We acknowledge Professor Eduardo Sontag for helpful advice. We especially acknowledge Dr. Yutaka Hori for helpful advice as well as detailed comments on the manuscript. We also thank Reviewer 3 for very detailed feedback.

\section{REFERENCES}

[1] E. Lubeck and L. Cai, "Single-cell systems biology by super-resolution imaging and combinatorial labeling," Nature Methods, vol. 9, no. 7, pp. 743-748, 2012.
[2] A. Raj, P. van den Bogaard, S. A. Rifkin, A. van Oudenaarden, and $\mathrm{S}$. Tyagi, "Imaging individual mRNA molecules using multiple singly labeled probes," Nature Methods, vol. 5, no. 10, pp. 877-879, 2008.

[3] J. W. Young, J. C. W. Locke, A. Altinok, N. Rosenfeld, T. Bacarian, P. S. Swain, E. Mjolsness, and M. B. Elowitz, "Measuring single-cell gene expression dynamics in bacteria using fluorescence time-lapse microscopy," Nature Protocols, vol. 7, no. 1, pp. 80-88, 2012.

[4] M. B. Elowitz, A. J. Levine, E. D. Siggia, and P. S. Swain, "Stochastic gene expression in a single cell," Science, vol. 297, no. 5584, pp. 1183-1186, 2002.

[5] A. Eldar and M. B. Elowitz, "Functional roles for noise in genetic circuits," Nature, vol. 467, no. 7312, pp. 167-173, 2010.

[6] J. Paulsson, "Models of stochastic gene expression," Physics of Life Reviews, vol. 2, no. 2, pp. 157-175, 2005.

[7] K. Sachs, O. Perez, D. Pe'er, D. A. Lauffenburger, and G. P. Nolan, "Causal protein-signaling networks derived from multiparameter single-cell data," Science, vol. 308, no. 5721, pp. 523-529, 2005.

[8] D. Husmeier, "Sensitivity and specificity of inferring genetic regulatory interactions from microarray experiments with dynamic Bayesian networks," Bioinformatics, vol. 19, no. 17, pp. 2271-2282, 2003.

[9] D. T. Gillespie, "Exact stochastic simulation of coupled chemical reactions," The Journal of Physical Chemistry, vol. 81, no. 25, pp. 2340-2361, 1977.

[10] T. Katayama, Subspace Methods for System Identification. Springer, 2005.

[11] B. Munsky and M. Khammash, "Identification from stochastic cellto-cell variation: a genetic switch case study," IET Systems Biology, vol. 4, no. 6, pp. 356-366, 2010.

[12] C. Zechner, J. Ruess, P. Krenn, S. Pelet, M. Peter, J. Lygeros, and H. Koeppl, "Moment-based inference predicts bimodality in transient gene expression," Proceedings of the National Academy of Sciences, vol. 109, no. 21, pp. 8340-8345, 2012.

[13] G. Lillacci and M. Khammash, "The signal within the noise: efficient inference of stochastic gene regulation models using fluorescence histograms and stochastic simulations," Bioinformatics, vol. 29, no. 18, pp. 2311-2319, 2013.

[14] O. Banerjee, L. El Ghaoui, A. d'Aspremont, and G. Natsoulis, "Convex optimization techniques for fitting sparse gaussian graphical models," in Proceedings of the 23rd International Conference on Machine Learning, 2006, pp. 89-96.

[15] A. P. Dempster, "Covariance selection," Biometrics, vol. 28, no. 1, pp. $157-175,1972$.

[16] T. K. Huang and J. G. Schneider, "Learning auto-regressive models from sequence and non-sequence data," in Advances in Neural Information Processing Systems 24, 2011, pp. 1548-1556.

[17] D. Hayden, Y. Yuan, and J. Goncalves, "Network reconstruction from intrinsic noise: Minimum-phase systems," in American Control Conference (ACC), 2014, pp. 4391-4396.

[18] E. Yeung, J. Kim, J. Goncalves, and R. M. Murray, "Global dynamical structure reconstruction from reconstructed dynamical structure subnetworks: Applications to biochemical reaction networks," to appear in the Proceedings of the 2015 IEEE Conference on Decision and Control, 2015.

[19] B. D. O. Anderson, "The inverse problem of stationary covariance generation," Journal of Statistical Physics, vol. 1, no. 1, pp. 133-147, 1969.

[20] K. Glover, "Structural aspects of system identification," Ph.D. dissertation, Massachusetts Institute of Technology, 1973.

[21] K. Zhou, J. C. Doyle, and K. Glover, Robust and Optimal Control. Prentice Hall, 1996.

[22] A. Rantzer, "On the Kalman-Yakubovich-Popov lemma," Systems \& Control Letters, vol. 28, no. 1, pp. 7-10, 1996.

[23] C. T. Lin, "Structural controllability," Automatic Control, IEEE Transactions on, vol. 19, no. 3, pp. 201-208, 1974. 